\title{
System of space - frequency filtering of linear and circular birefringence in cancer diagnosis
}

\author{
Koval G.D., Raranskiy M.D. \\ Optics and Spectroscopy Department, \\ Chernivtsi National University, \\ 2 Kotsybinsky Str., Chernivtsi, 58012 Ukraine \\ yuriyu@gmail.com
}

\begin{abstract}
The optical model of polycrystalline networks of histological sections of rectum wall is suggested. The results of investigating the interrelation between the values of statistical (statistical moments of the $1^{\text {st }}-4^{\text {th }}$ order) parameters are presented. They characterize the coordinate distributions of the fourth parameter of Stokes vector of Fourier transforms of laser images of rectum wall histological sections and oncological changes. The diagnostic criteria of rectum cancer are determined.
\end{abstract}

Key words: Fourier-stokes polarimetry, microscopic images, polycrystalline networks, polarizationally sensitive optical coherent tomography, histological sections of rectum wall biopsy

\section{Introduction}

A new direction of optical physical diagnostics [1-8] - laser polarimetry of microscopic images of polycrystalline networks of biological tissues histological sections has been developed in recent years. This approach is focused on in vitro measurements of coordinate distributions of Stokes vector parameters of biological tissues laser images with further complex (statistical, correlation, fractal, singular, wavelet, etc.) analysis on the basis of the obtained data arrays [9-15].

Another diagnostic biomedical laser technique - polarizationally sensitive optical coherent tomography - also became widely spread [10]. It is based on measuring the parameters of Stokes vector in the remote (Fourier) diffraction zone of the field of scattered laser radiation.

That is why the task of combining the information potentiality of the above mentioned techniques of optical diagnostics basing on the development of new methods of analysis and processing of polarizationally inhomogeneous images of biological tissues in the Fourier plane of scattered radiation field proves to be topical.

This research is focused on the development of experimental technique of polarization investigation of coordinate distributions of Stokes vector parameters of the laser field in the Fourier plane for diagnostics and differentiation of the severity of pathological changes in rectum tissue biopsy.

\section{Basic Analytical Relations}

It was determined for the fields of scattered laser radiation that polarization state in every point with $(n, m)$ coordinates is formed by the mechanisms of statistical interference and is determined using the following relations

$$
\begin{aligned}
& \alpha(n, m)=0,5 \arcsin \left[\frac{\sin 2 \Theta(n, m)}{\cos \delta(n, m)}\right] ; \\
& \beta(n, m)=0,5 \operatorname{arctg}\left[\frac{\sin \delta(n, m)}{\cos 2 \Theta(n, m)}\right],
\end{aligned}
$$

where $\Theta=\operatorname{arctg} \frac{E_{y}}{E_{x}}-$ the phase angle, $\delta$ - phase shift between the orthogonal components $E_{x}, E_{y}$ of laser radiation amplitude determined from relations 


$$
\begin{aligned}
& E_{x} e^{i \delta_{x}}=e^{i \delta_{0 x}} E_{0 x}\left[\left(\cos ^{2} \rho+e^{-i \varphi} \sin ^{2} \rho\right) \sin ^{2} \theta\right]+e^{i \delta_{0 y}} E_{0 y}\left[\cos \rho \sin \rho\left(1-e^{-i \varphi}\right) \cos ^{2} \theta\right] ; \\
& E_{y} e^{i \delta_{y}}=e^{i \delta_{0 x}} E_{0 x}\left[\cos \rho \sin \rho\left(1-e^{-i \varphi}\right) \sin ^{2} \theta\right]+e^{i \delta_{0 y}} E_{0 y}\left[\left(\cos ^{2} \rho+e^{-i \varphi} \sin ^{2} \rho\right) \cos ^{2} \theta\right] .
\end{aligned}
$$

Here $\delta_{0 x}, \delta_{0 x}$ - phases of orthogonal components $\left(E_{0 x}, E_{0 y}\right)$ of the amplitude of the laser image probing polycrystalline network; $\rho \varphi \theta \quad \delta=\delta_{x}-\delta_{y}=\varphi+\theta$.

Expressions (3) and (4) are "input" parameters for diffraction integrals determining further diffractioninterferential process of evolution of amplitude-phase distributions of object field.

For the case of finding the biological tissue layer in the focal plane of the objective the following can be written

$$
\begin{gathered}
U_{x}\left(m^{*}, n^{*}\right)=\frac{A}{i \lambda f} \int_{-\infty}^{+\infty} E_{x}(m, n) \exp \left[-i \frac{2 \pi}{\lambda f}\left(n n^{*}+m m^{*}\right) d n d m\right] ; \\
U_{y}\left(m^{*}, n^{*}\right)=\frac{A}{i \lambda f} \int_{-\infty}^{+\infty} E_{y}(m, n) \exp \left[-i \frac{2 \pi}{\lambda f}\left(n n^{*}+m m^{*}\right) d n d m\right] .
\end{gathered}
$$

Here $f$ - focus distance of the objective; $\lambda$ - wave length of laser radiation; $n, m$ and $n^{*}, m^{*}$ - coordinates of the points in the image plane and Fourier plane respectively.

Diffraction integrals (5) and (6) enable to determine the asymmetry degree of Fourier spectrum in two mutually transverse directions

$$
\Delta(v)=\frac{U_{x}\left(m^{*}\right)+U_{y}\left(m^{*}\right)}{U_{x}\left(n^{*}\right)+U_{y}\left(n^{*}\right)},
$$

where

$$
\begin{gathered}
U_{x}\left(m^{*}\right)=\frac{A}{i \lambda f} \int_{-\infty}^{+\infty} E_{x}(m) \exp \left[-i \frac{2 \pi}{\lambda f} m m^{*}\right] d m=\frac{A}{i \lambda f} \int_{-\infty}^{+\infty} E_{x}(m) \exp \left[-i 2 \pi m v_{m}\right] d m ; \\
U_{x}\left(n^{*}\right)=\frac{A}{i \lambda f} \int_{-\infty}^{+\infty} E_{x}(m) \exp \left[-i \frac{2 \pi}{\lambda f} n n^{*}\right] d n=\frac{A}{i \lambda f} \int_{-\infty}^{+\infty} E_{x}(m) \exp \left[-i 2 \pi n v_{n}\right] d n \\
U_{y}\left(m^{*}\right)=\frac{A}{i \lambda f} \int_{-\infty}^{+\infty} E_{y}(m) \exp \left[-i \frac{2 \pi}{\lambda f} m m^{*}\right] d m=\frac{A}{i \lambda f} \int_{-\infty}^{+\infty} E_{y}(m) \exp \left[-i 2 \pi m v_{m}\right] d m ; \\
U_{y}\left(n^{*}\right)=\frac{A}{i \lambda f} \int_{-\infty}^{+\infty} E_{y}(n) \exp \left[-i \frac{2 \pi}{\lambda f} n n^{*}\right] d n=\frac{A}{i \lambda f} \int_{-\infty}^{+\infty} E_{y}(n) \exp \left[-i 2 \pi n v_{n}\right] d n
\end{gathered}
$$
written

Thus, taking into account relations (1)-(6) the following expressions for the Stokes vector parameters can be 


$$
\begin{gathered}
S_{1}\left(m^{*}, n^{*}\right)=E_{x} E_{x}^{*}+E_{y} E_{y}^{*} ; \\
S_{2}\left(m^{*}, n^{*}\right)=E_{x} E_{x}^{*}-E_{y} E_{y}^{*} ; \\
S_{3}\left(m^{*}, n^{*}\right)=E_{x} E_{y}^{*}-E_{y} E_{x}^{*} ; \\
S_{4}\left(m^{*}, n^{*}\right)=i\left(E_{y} E_{x}^{*}-E_{x} E_{y}^{*}\right),
\end{gathered}
$$

and asymmetry degree of $S_{k=1 ; 2 ; 3 ; 4}\left(m^{*}, n^{*}\right)$ distributions can be determined та визначити ступень асиметрії (relations (7) - (12)).

For objective (quantitative) estimation of distributions $S_{k=1 ; 2 ; 3 ; 4}\left(m^{*}, n^{*}\right)$ the statistical, correlation and spectral approaches were used.

\subsection{Statistical Approach}

Statistical moments of the first $\left(R_{1}\right)$, second $\left(R_{2}\right)$, third $\left(R_{3}\right)$ and fourth $\left(R_{4}\right)$ orders were calculated by the following algorithms

$$
\begin{gathered}
R_{1}=\frac{1}{P} \sum_{j=1}^{P}\left(\left|S_{k}\right|\right)_{j} ; \\
R_{2}=\sqrt{\frac{1}{P} \sum_{j=1}^{P}\left(S_{k}^{2}\right)_{j} ;} \\
R_{3}=\frac{1}{R_{2}^{3}} \frac{1}{P} \sum_{j=1}^{P}\left(S_{k}^{3}\right)_{j} ; \\
R_{4}=\frac{1}{R_{2}^{2}} \frac{1}{P} \sum_{j=1}^{P}\left(S_{k}^{4}\right)_{j},
\end{gathered}
$$

where $P=800 \times 600$ - total amount of pixels of CCD-camera recording the polarizationally-inhomogeneous object field in Fourier plane.

\subsection{Correlation Approach}

One of the most well-known and effective approaches to the analysis of the coordinate structure of $S_{k=1 ; 2 ; 3 ; 4}\left(m^{*}, n^{*}\right)$ distributions consists in their autocorrelation comparison in two mutually transverse directions of CCD-camera plane using the next algorithms

$$
\begin{aligned}
& \mathrm{K}\left(\Delta v_{x}\right)=\frac{1}{\left(v_{\max }\right)_{x}} \sum_{j=1}^{N} S_{k}\left(v_{x}\right) S_{k}\left(v_{x}-j \Delta v_{x}\right) ; \\
& \mathrm{K}\left(\Delta v_{y}\right)=\frac{1}{\left(v_{\max }\right)_{y}} \sum_{j=1}^{M} S_{k}\left(v_{y}\right) S_{k}\left(v_{y}-j \Delta v_{y}\right) .
\end{aligned}
$$

Here $\left(\Delta v_{x}, \Delta v_{y}\right)$ are "steps", with which spatial-frequency coordinates $\left(v_{x}, v_{y}\right)$ change.

For two mutually transverse directions $n^{*}, m^{*}$ in Fourier plane these parameters are determined as $v\left(n^{*}\right)=\frac{k n_{0}^{*}(1 p i x)}{\lambda f}$ and $v\left(m^{*}\right)=\frac{k m_{0}^{*}(1 p i x)}{\lambda f}$ respectively. 
In order to estimate the coordinate homogeneities or, vice versa, heterogeneities (asymmetry) of Fourier images of $S_{k=1 ; 2 ; 3 ; 4}\left(m^{*}, n^{*}\right)$ distributions the following algorithm of autocorrelation dependencies (15) and (16) processing were used

$$
\begin{aligned}
& d Q_{1}=R_{1}\left(\mathrm{~K}\left(\Delta v_{y}\right)\right) / R_{1}\left(\mathrm{~K}\left(\Delta v_{x}\right)\right) ; \\
& d Q_{2}=R_{2}\left(\mathrm{~K}\left(\Delta v_{y}\right)\right) / R_{2}\left(\mathrm{~K}\left(\Delta v_{x}\right)\right) ; \\
& d Q_{3}=R_{3}\left(\mathrm{~K}\left(\Delta v_{y}\right)\right) / R_{3}\left(\mathrm{~K}\left(\Delta v_{x}\right)\right) ; \\
& d Q_{4}=R_{4}\left(\mathrm{~K}\left(\Delta v_{y}\right)\right) / R_{4}\left(\mathrm{~K}\left(\Delta_{x}\right)\right),
\end{aligned}
$$

where $R_{1 ; 2 ; 3 ; 4}$ are statistical moments of the $1^{\text {st }}-4^{\text {th }}$ orders (relations (14)) of values distributions of autocorrelation dependencies $\mathrm{K}\left(\Delta v_{x}\right)$ and $\mathrm{K}\left(\Delta v_{y}\right)$.

Further, the set of values $d Q_{k=1 ; 2 ; 3 ; 4}$ (relation (17)) will be called "cross-correlation" moments of the $1^{\text {st }}-4^{\text {th }}$ orders.

\subsection{Spectral Approach}

Statistical analysis of power spectra $W(\mathrm{~K}(\Delta v))$ of the Stokes vector parameters $S_{k=1 ; 2 ; 3 ; 4}\left(m^{*}, n^{*}\right)$ distribution consists in the following procedure:

- $\quad$ power spectra $W(\mathrm{~K}(\Delta v))$ for two mutually transverse directions were calculated

$$
\begin{aligned}
& W\left(v_{x}\right)=\frac{1}{N} \int_{0}^{N} \mathrm{~K}\left(\Delta v_{x}\right) \exp \left[-\left(\frac{2 \pi}{v_{x} x}\right)\right] d x ; \\
& W\left(v_{y}\right)=\frac{1}{N} \int_{0}^{N} \mathrm{~K}\left(\Delta v_{y}\right) \exp \left[-\left(\frac{2 \pi}{v_{y} y}\right)\right] d y ;
\end{aligned}
$$

- Log - $\log$ dependencies of power spectra $\log W-\log (v)$ were calculated;

- for dependencies $\log W-\log (v)$ the ensemble of statistical moments of the $1^{\text {st }}-4^{\text {th }}$ orders was determined

$$
\begin{aligned}
& d F_{1}=R_{1}\left(W\left(\Delta v_{y}\right)\right) / R_{1}\left(W\left(\Delta v_{x}\right)\right) ; \\
& d F_{2}=R_{2}\left(W\left(\Delta v_{y}\right)\right) / R_{2}\left(W\left(\Delta v_{x}\right)\right) ; \\
& d F_{3}=R_{3}\left(W\left(\Delta v_{y}\right)\right) / R_{3}\left(W\left(\Delta v_{x}\right)\right) ; \\
& d F_{4}=R_{4}\left(W\left(\Delta v_{y}\right)\right) / R_{4}\left(W\left(\Delta v_{x}\right)\right) ;
\end{aligned}
$$

Hereinafter the set $d F_{k=1 ; 2 ; 3 ; 4}$ (relation (14)) will be called "cross-spectral" moments of the $1^{\text {st }}-4^{\text {th }}$ orders. 


\section{Optical Realization of Fourier Transform of Polarizationally Inhomogeneous Laser Fields}

Histological sections of biological tissues samples were located in the focal plane of microobjective (focal distance $f=450 \mu \mathrm{m}$, magnification $4 \mathrm{X}$ ) were projected onto the plane of light sensitive plate (800x600 pixels) of CCDcamera $(P=m \times n=600$ pix $\times 800$ pix $)$, which was placed at focal distance to assure the measurement range of structural elements of Fourier spectrum of biological tissues image for further geometrical sizes $2 \mu \mathrm{m}-2000 \mu \mathrm{m}$.

The analysis of polarization structure of Fourier spectra of the images of biological tissues histological sections was performed by means of polarizer and quarter-wave plate.

The technique of measuring Stokes vector $S_{k}$ parameters is described in detail in [10 - 14] and corresponds to the following algorithm

$$
S=\left\{\begin{array}{c}
\left(\begin{array}{c}
1 ; \\
\left.I_{0}-I_{90} / I_{0}+I_{90}\right)
\end{array}\right) ; \\
\left(I_{45}-I_{135} / I_{0}+I_{90}\right) \\
\left(I_{\otimes}-I_{\oplus} / I_{0}+I_{90}\right)
\end{array}\right\} .
$$

Here $I_{0}, I_{90}, I_{45}, I_{135}$ are intensities of plane-polarized components with azimuths $0^{0}, \quad 90^{0}, 45^{0}, 135^{0} ; I_{\otimes}, \quad I_{\oplus}$ are counterclockwise $(\otimes)$ and clockwise $(\oplus)$ circularly polarized components of laser radiation.

\section{Diagnostic Potentiality of Fourier Stokes Polarimetry of Laser Fields of Biological Tissues Histological Sections in vitro}

Histological sections of rectum wall biopsy with benign (group of samples 1-9) and malignant (group of samples 2-10) were used as objects of investigation.

This research is confined to the analysis of distributions structure of the $4^{\text {th }}$ Stokes vector parameter most vividly characterizing the changes in optical anisotropy of polycrystalline biological networks.

The coordinate (left parts) and probability (right parts) distributions of the values of the $4^{\text {th }}$ Stokes vector parameter of optically realized Fourier spectrum of laser radiation scattered by histological sections of rectum wall biopsy of group 2 (Fig. 1) and group 2 (Fig. 2) are presented in Fig. 1 and Fig. 2.
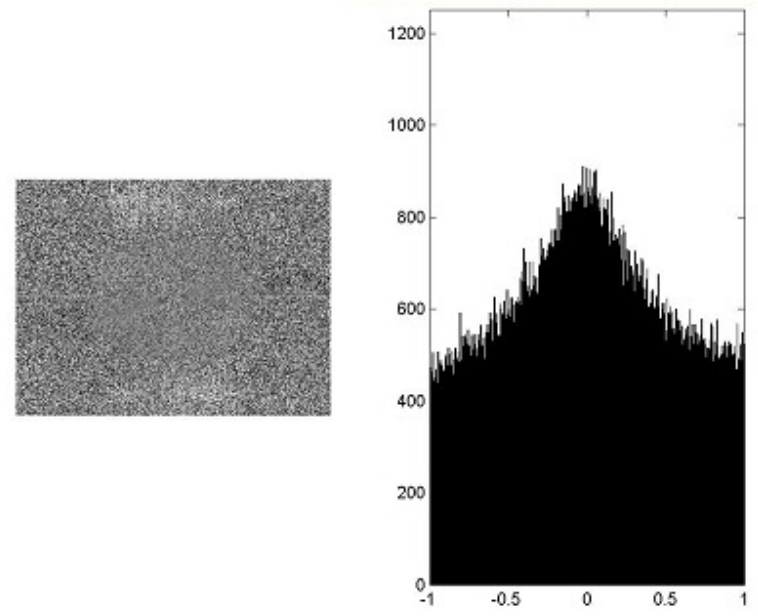

(a)

(b)

Figure 2. Coordinate structure and histogram of values distribution of the $4^{\text {th }}$ parameter of Stokes vector scattered by histological section of group 1 in Fourier plane. 

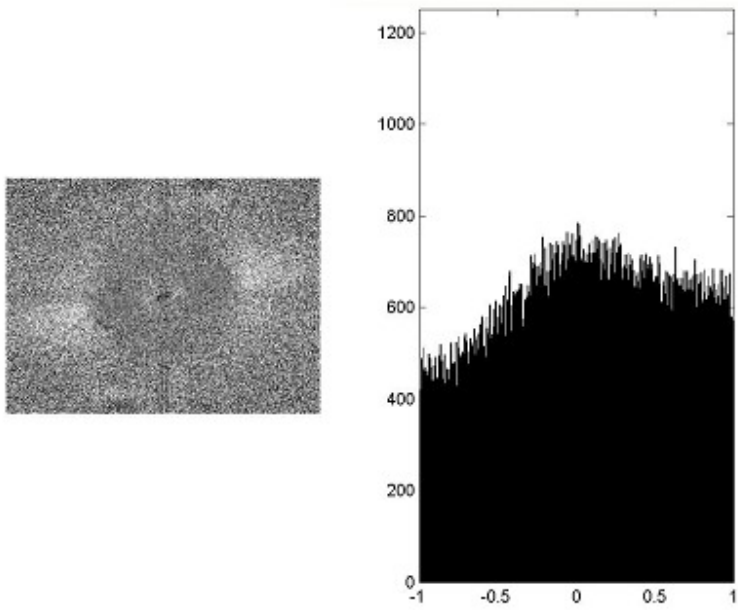

(a)

(b)

Figure 2. Coordinate structure and histogram of values distribution of the $4^{\text {th }}$ parameter of Stokes vector scattered by histological section of group 2 in Fourier plane.

The obtained data show that the value of the $4^{\text {th }}$ Stokes vector parameter in different points of Fourier spectrum of the field of scattered laser radiation irrespective of the type of pathological changes of rectum tissue undergoes maximal theoretically possible range of changes $-1,0 \leq S_{4} \leq+1,0$. This fact indicates the wide experimentally realized change range $\left(0^{0} \leq \varphi, \theta \leq 180^{0}\right)$ of phase shifts between orthogonal amplitude components of the laser beam transformed by optically anisotropic network of rectum wall tissue.

The main peculiarity of probability distributions $S_{4}(m \times n)$ consists in the asymmetric structure of corresponding histograms. This feature is connected with a large number of maximal phase shifts $\left(\varphi, \theta \rightarrow \max \Leftrightarrow S_{4} \rightarrow \min \right)$ due to interferential mechanism (the change of phase shifts between the orthogonal amplitude components) of forming phaseinhomogeneous structure of laser radiation field in Fourier plane.

The results of the $1^{\text {st }}-4^{\text {th }}$ order moments calculation (relation (14)) characterizing the coordinate distributions $\left(\begin{array}{ccc}\left(S_{4}\right)_{11} & \ldots & \left(S_{4}\right)_{1 n} \\ \cdot & \ldots & \cdot \\ \left(S_{4}\right)_{m 1} & \rightarrow & \left(S_{4}\right)_{m n}\end{array}\right)$ (relation $\left.(21)\right)$ in Fourier plane of the field of laser radiation transformed by histological sections of rectum wall with benign (group 1) and malignant (group 2) tumors are presented in Table 1.

Table 1. Statistical moments of the $1^{\text {st }}-4^{\text {th }}$ orders of Fourier spectrum $S_{4}$

\begin{tabular}{|c|c|c|}
\hline Parameters & Benign changes & Malignant changes \\
\hline$R_{1}$ & $0,28 \pm 0,0012$ & $0,07 \pm 0,021$ \\
\hline$R_{2}$ & $0,11 \pm 0,008$ & $0,39 \pm 0,014$ \\
\hline$R_{3}$ & $1,14 \pm 0,009$ & $0,59 \pm 0,016$ \\
\hline$R_{4}$ & $2,47 \pm 0,017$ & $0,79 \pm 0,027$ \\
\hline
\end{tabular}

It was determined that the difference between the statistical parameters $R_{j=1 ; 2 ; 3 ; 4}$ for both groups of histological sections amounts to: mean $R_{1}-4$ times; dispersion $R_{2}-3.8$ times; asymmetry $R_{3}-1.9$ times; excess $R_{4}-3.1$ times. On the basis of this the following parameters of sensitivity and specificity in differentiation of benign and malignant changes of rectum wall: 
Group $1(q=9)$

\begin{tabular}{|c|c|c|}
\hline State & Benign changes & Malignant changes \\
\hline Parameters & $b=7$ & $d=2$ \\
\hline
\end{tabular}

Group $2(q=10)$

\begin{tabular}{|c|c|c|}
\hline State & Benign changes & Malignant changes \\
\hline Parameters & $c=3$ & $a=6$ \\
\hline
\end{tabular}

Thus, sensitivity $S e$ and specificity $S p$ equal to: $\operatorname{Se}\left(d Q_{2}\right)=78 \% ; \operatorname{Sp}\left(d Q_{4}\right)=77 \%$.

\section{Conclusions}

1. The complex technique of estimating polarizationally inhomogeneous field of scattered laser radiation in Fourier plane is suggested. It is based on determination of values and range of changes of statistical moments of the $1^{\text {st }}$ $4^{\text {th }}$ orders, characterizing the probability, coordinate and spatial-frequency structure of Stokes vector parameters coordinate distributions.

2. The ensemble of diagnostically urgent statistical (statistical moments of the $1^{\text {st }}-4^{\text {th }}$ orders), parameters of coordinate distributions of the $4^{\text {th }}$ Stokes vector parameter of Fourier image of polarizationally inhomogeneous image of optically anisotropic component of histological sections of stomach wall tissue is determined. By means of these parameters differentiation of benign and malignant tumor with high sensitivity and specificity levels is realized for the first time.

\section{References}

[1] W. F. Cheong, S. A. Prahl, A.J. Welch, "A review of the optical properties of biological tissues", IEEE J. Quantum Electr., Vol. 26, P. 2166-2185, (1990).

[2] "Quantitative Spectroscopy in Tissue “, Ed. by K. Frank, M. Kessler. - Frankfurt am Main: pmiVerlag, (1992).

[3] Moseley, H., "Special Issue on Optical Radiation Technique in Medicine and Biology", Phys. Med. Biol., Vol. 24., P. 759-996, (1997).

[4] "Light scattering spectroscopy of epithelial tissues: principles and applications", ed. by V.V. Tuchin, Bellingham : SPIE Press, (2002).

[5] Mourant, J.R., Freyer, J. P., Hielscher, A. H., « Mechanisms of light scattering from biological cells relevant to noninvasive optical-tissue diagnosis", Appl. Opt., Vol. 37, P. 3586-3593, (1998).

[6] Drezek, R., Dunn, A., Richards-Kortum, R., "Light scattering from cells: finite-difference time-domain simulations and goniometric measurements", Appl. Opt., Vol. 38, P. 3651-3661, (1999).

[7] Backman, V., Wallace, M., Perelman, L. T., "Diagnosing Cancers Using Spectroscopy", Nature,Vol. 405, (2000).

[8] Perelman, L. T., Bachman, V., Wallace, M., "Observation of periodic fine structure in reflectance from biological tissue: a new technique for measuring nuclear size distribution", Phys. Rev. Lett., Vol. 80, P. 627630, (1998).

[9] Angelsky, O.V., Ushenko, A.G., Ushenko, Yu.A., Ushenko, Ye.G., "Polarization singularities of the object field of skin surface", Journal of Physics D: Applied Physics, 39 (16), art. no. 005, pp. 3547-3558, (2006).

[10] Angelsky, O.V., Demianovsky, G.V., Ushenko, A.G., Burkovets, D.N., Ushenko, Yu.A., "Wavelet analysis of two-dimensional birefringence images of architectonics in biotissues for diagnosing pathological changes", Journal of Biomedical Optics, 9 (4), pp. 679-690, (2004).

[11] Angel'skiî, O. V., Ushenko, A. G., Arkhelyuk, A. D., Ermolenko, S. B., Burkovets, D. N., Ushenko, Yu. A., "Laser polarimetry of pathological changes in biotissues", Optika i Spectroskopiya, 89( 6), 1050-1055, (2000).

[12] O.V.Angelsky, P.P.Maksimyak, T.O.Perun, "Dimensionality in optical fields and signals", Applied Optics, v.32, No.30, pp.6066-6071, (1993).

[13] A. Y. Bekshaev, O. V. Angelsky, S. G. Hanson, and C. Y. Zenkova, "Scattering of inhomogeneous circularly polarized optical field and mechanical manifestation of the internal energy flows", Phys. Rev. A 86, 023847, (2012). 
[14] Angel'skii, O.V., Ushenko, O.G., Burkovets, D.N., Arkhelyuk, O.D., Ushenko, Yu.A. "Polarization-correlation studies of multifractal structures in biotissues and diagnostics of their pathologic changes", Laser Physics, 10 (5), pp. 1136-1142, (2000).

[15] Angelsky, O.V., Besaha, R.N., Mokhun, I.I., "Appearance of wave front dislocations under interference among beams with simple wave fronts", Optica Applicata, Volume 27, Issue 4, Pages 272-278, (1997). 